\title{
IMPROVED TONE RECOGNITION FOR FLUENT MANDARIN SPEECH BASED ON NEW INTER-SYLLABIC FEATURES AND ROBUST PITCH EXTRACTION
}

\author{
Wan-yi Lin and Lin-shan Lee \\ Graduate Institute of Communication Engineering, National Taiwan University \\ Taipei, Taiwan, Republic of China \\ mfan@speech.ee.ntu.edu.tw, Islee@gate.sinica.edu.tw
}

\begin{abstract}
ABSTRCT
Tone recognition for fluent Mandarin speech has always been a very difficult problem, because the pitch contours vary seriously with the context conditions and the complicated tone behavior is difficult to analyze. In this paper, a new set of four inter-syllabic features are identified to characterize quantitatively such pitch contour variation with respect to the context conditions. In addition, a robust pitch extraction method is proposed by integrating the Adaptive Gabor Representation (AGR) and Instantaneous Frequency Amplitude Spectrum (IFAS). Experimental results indicate that accurate pitch values can be extracted under various noisy conditions, and the tone recognition accuracy can be improved significantly.
\end{abstract}

\section{INTRODUCTION}

Chinese is a typical tonal language. Each Chinese character is pronounced as a monosyllabe. There is a total of about 416 phonologically allowed syllables in Mandarin Chinese, if the difference in tones are disregarded. But if the differences in tones are considered, there are about 1345 syllables. The tones in syllables have lexical meanings in Chinese; as a result the tone recognition plays an important role in Chinese speech recognition. There exist four lexical tones (tone 1 to tone 4) plus a neutral tone (tone 5) in Mandarin. These numbers are slightly different for different dialects. The most significant difference among the tones is expressed in the shapes of pitch contours, so we need to have an accurate enough pitch estimation method in order to perform tone recognition.

Many algorithms for pitch estimation have been studied extensively ever since many years ago, some of which utilized the various characteristics of speech signals in time domain, some in frequency domain, and some in both [1]. The performance of most of the algorithms is satisfactory for clean speech, but degrades rapidly under noisy conditions. As robust speech recognition in adverse environment becomes highly desired in recent years, accurate tone recognition which has to be based on accurate pitch estimation under noisy conditions become more and more important in Chinese speech recognition.

Substantial efforts have made in tone recognition for Chinese syllables, even including that for Cantonese, [2], although the major efforts were for Mandarin. For Mandarin, tone 5, the neutral tone, appears slightly less frequent than the four lexical tones and is often seriously confused with the four lexical tones. Some of the studies only tried to recognize the four lexical tones. Vector quantization, HMM, and neural networks have been applied for tone recognition over since roughly fifteen years ago $[3,4]$. The context dependency [5] as well as the tone nuclei have also been introduced [6].

In this paper, we propose a new method integrating the Adaptive Gabor Expansion (AGR) for signal analysis and the Instantaneous Frequency (IF) for accurate pitch estimation under noisy environment. Although AGR have been used with WignerVillie Distribution (WVD) to obtain the spectrogram of speech signals, [7], the fewer number of the frequency components in WVD spectrogram may also make the important harmonic components missed or defocused. This may degrade the AGR performance under non-Gaussian noise environment. So we used the Instantaneous Frequency (IF) to analyze the speech frames after AGR. In this way the harmonic components can be emphasized. Significant improvements in pitch estimation accuracy under serious noisy conditions were observed in the experiments.

There have been two major difficulties in Mandarin tone recognition: the pitch contours of neutral tone vary significantly and even irregularly, and the pitch contours for all the different tones are seriously context dependent, or affected by adjacent syllables. In this paper, we propose four inter-syllabic features to better model these two phenomena. Preliminary experiments showed that with these four extra features, the recognition accuracy for the neutral tone can be significantly improved while confusion among different tones can be largely reduced.

The organization for the rest of this paper is as follows. In section 2 we present the new pitch extraction method. While the inter-syllabic features for tone recognition are discussed in section 3. The experimental results are then described and analyzed in section 4 , and the conclusions are made in section 5 .

\section{PITCH EXTRACTION}

In this section, we propose a new pitch extraction method. Adaptive Gabor Representation (AGR) [8] is first used to analyze the speech signal; and then the signal is transformed into 
Instantaneous Frequency Amplitude Spectrum (IFAS) to emphasis the harmonic components. Detailed tracking and smoothing then follows. The complete pitch extraction flowchart is shown in Fig.1.

\subsection{Adaptive Gabor Representation [8]}

The adaptive representation is usually used to find the most important factors that characterize the signals which we are interested. It flexibly decomposes a signal, $s(t)$, into a linear expansion of waveforms selected from a dictionary of redundant elementary functions, $D=\left\{h_{p}(t)\right\}$, for all $p$

$$
s(t)=\sum_{p} B_{p} h_{p}(t)
$$

where $B_{p}$, all $\mathrm{p}$, is a set of proper coefficients. Because the Gaussian-type signal achieves the lower bound of the uncertainty inequality, it is a natural selection for the elementary functions used for adaptive representation discussed here, that is,

$$
h_{p}(t)=\left(\frac{\alpha_{p}}{\pi}\right)^{\frac{1}{4}} \exp \left\{-\frac{\alpha_{p}}{2}\left(t-T_{p}\right)^{2}\right\} \exp \left\{j \Omega_{p} t\right\}
$$

where $\left(T_{p}, \Omega_{p}\right)$ is the time-frequency center of the elementary function $h_{p}(t)$, and $\alpha_{p}{ }^{-1}$ is the variance of the Gaussian function. So $h_{p}(t)$ in equation (2) is specified by three parameters: $T_{p}, \Omega_{p}$, and $\alpha_{p}$. The adaptive representation based on these elementary functions $h_{p}(t)$ is so-called Adaptive Gabor Representation (AGR).

The best set of $h_{p}(t)$ can be selected iteratively, starting with $h_{p}(t)$ and then increasing the value of $\mathrm{p}$ one by one as the following,

$$
s_{p}(t)=s_{p-1}(t)-B_{p-1} h_{p-1}(t)
$$

where the best set of $\left(T_{p}, \Omega_{p}\right)$, however, can be obtained with the following expression, which tries to maximize the inner product between $s_{p}(t)$ and $h_{p}(t)$ by discretizing $T_{p}$ and $\Omega_{p}$ into small intervals of time and frequency,

$$
\begin{aligned}
\left|B_{p}\right|^{2} & =\max _{h_{p}}\left|\left\langle s_{p}(t), h_{p}(t)\right\rangle\right|^{2} \\
& =\max _{i_{p}} \mid \sum_{i} s_{p}\left[\left.i\left(\frac{\alpha_{p}}{\pi}\right)^{\frac{1}{4}} \exp \left\{-\frac{\alpha_{p}}{2}\left(i-m \Delta M_{p}\right)\right\} \exp \left\{-j \frac{2 m \Delta N_{p}}{L} i\right\}\right|^{2}\right.
\end{aligned}
$$

where $\Delta M_{p}$ and $\Delta N_{p}$ denote the time and frequency intervals, and $\mathrm{L}$ denotes the effective length of the Gaussian function $h_{p}(t)$ with the largest variance.

When $\alpha_{p}$ is smaller, equation (4) has a poor time resolution and is very sensitive to the change of $\Delta N_{p}$. However, a nice property of equation (4) is that at a fixed $\alpha_{p}$, it is a regular shorttime Fourier transform. So we can estimate $\left(T_{p}, \Omega_{p}, \alpha_{p}\right)$ by first setting a small $\alpha_{p}$, a small $\Delta N_{p}$, and a large $\Delta M_{p}$ then

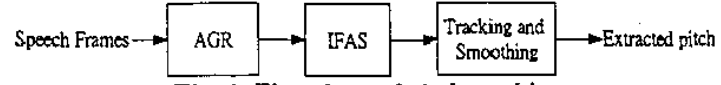

Fig. 1: Flowchart of pitch tracking

searching through all $\mathrm{m}$ and $\mathrm{n}$ to obtain $\left(T_{p}=\mathrm{m}^{0} \Delta M_{p}\right.$, $\left.\Omega_{p}=\mathrm{n}^{0} \Delta N_{p}\right)$ which maximize $\left|B_{p}\right|^{2}$. We can then set $\Delta M_{p}$, smaller, and search for $\mathrm{m}^{1}$ and $\mathrm{n}^{1}$ near $\mathrm{m}^{0}$ and $\mathrm{n}^{0}$. This procedure can be performed recursively until $\left|B_{p}\right|^{2}$ converges.

\subsection{Instantaneous Frequency Amplitude Spectrum}

After we represent the speech signal as the weighted sum of $h_{p}(t)$ as in section 2.1, and the Instantaneous Frequency (IF) of each component $B_{p} h_{p}(t)$ is then as the time derivative of the phase[9],

$$
\lambda\left(\Omega_{p}, t\right)=\frac{\partial}{\partial t} \arg \left[B_{p} h_{p}(t)\right]
$$

The Instantaneous Frequency Amplitude Spectrum (IFAS), $S\left(\lambda_{0}, t\right)$, can then be specified as the summation of the amplitudes of the component elementary functions for all $p$, over a set of intervals $\omega_{0}$ on the frequency axis $\omega_{0}=\left\{\Omega_{p} \mid \lambda_{0} \leq \lambda\left(\Omega_{p}, t\right) \leq \lambda_{0}+\Delta \lambda\right\}$, where $\Delta \lambda$ is some pre-defined instantaneous frequency interval. Then the IFAS, $S\left(\lambda_{0}, t\right)$, is defined as

$$
S\left(\lambda_{v}, t\right)=\lim _{\Delta \lambda \rightarrow 0} \frac{1}{\Delta \lambda} \sum_{p, \omega_{b}}\left(\frac{\alpha_{p}}{\pi}\right)^{\frac{1}{4}} \exp \left\{-\frac{\alpha_{p}}{2}\left(t-T_{p}\right)^{2}\right\}
$$

The IFAS, $S\left(\lambda_{0}, t\right)$, of a typical speech frame is shown in Fig. 2. The IF value with the maximal value of $S\left(\lambda_{0}, t\right)$ as obtained above is the pitch, but this value should not be too small. So we set a threshold $\eta$ for the ratio of this maximal value in IFAS to the frame energy. If the ratio is under threshold $\eta$, we assume this frame has no pitch.

\subsection{Tracking and Smoothing}

After finding the maximum in (6) on each frame of speech, we can obtain the estimated pitch for every frame, unless no pitch is found as mentioned above. Because the pitch curve of voiced speech is continuous locally, two steps for tracking and smoothing the pitch values to ensure the continuity of pitch contour were developed:

stepl. For every two adjacent frames, check if the difference between their pitch values is less than $12 \%$ of either one of these two frequencies. If two adjacent frames satisfy the above condition, their pitch values form a portion of one pitch contour. Otherwise the new pitch value will be deleted. This step will produce a set of piecewisecontinuous pitch contours. 


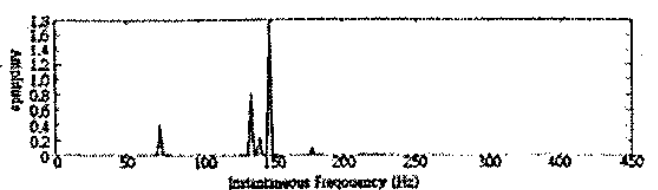

Fig.2: IFAS of a speech signal

step2. Check if the length of each continuous pitch contour formed in step one has more than five pitch values. If not, it is discarded.

\section{INTER-SYLLABIC FEATURES FOR TONE RECOGNITION}

It is well known that the properties of the tones are seriously affected by the context, or the tones of the preceding and following syllables. However, it is not easy to identify some features addressing such phenomenon. In this section, we proposed four inter-syllabic features; one is the ratio of duration of adjacent syllables, and other three are average $|\mathrm{dfO}|,|\mathrm{fO}|$, and maximum $\mathrm{fo}$ difference of a syllable.

\subsection{Duration Ratio for Modeling the Neutral Tone}

A well known characteristic of the neutral tone is that it is often produced relatively shortly and lightly. Unfortunately, these two characteristics are not strong enough to distinguish neutral tone from the others, because in many cases syllables with other tones may be produced equally shortly and lightly.

We observed that in order to produce a neutral-tone syllable clearly, the syllable before the neutral tone is almost always produced longer. So if the present syllable is of neutral tone, the ratio of the duration of the previous syllable to that of the present syllable is usually significantly higher than in other cases. Fig. 3(a) shows an example sentence randomly picked up from the HUB4 Mandarin broadcast news database, and the duration ratios for adjacent syllables for this sentence is shown in Fig. 3(b). It is clearly that the third syllable, /de $5 /$, is of the neutral tone and the preceding syllable, / chang $2 /$, is quite long. The ratio for this case, 1.43 , is much higher than that for other cases. It should be noted that the fourth syllable in the sentence, /shul/, is of tone 1 but almost as short as /de5/. This is why the duration alone can't be used as a feature for the neutral tone.

\subsection{Features Modeling Perturbations of Pitch Contours Caused by Adjacent Syllables}

As mentioned above, it is well known that the pitch contour of a syllable is perturbed seriously by the tone of its preceding and following syllables (the so-called tone sandhi phenomenon). We (a) Speech frame

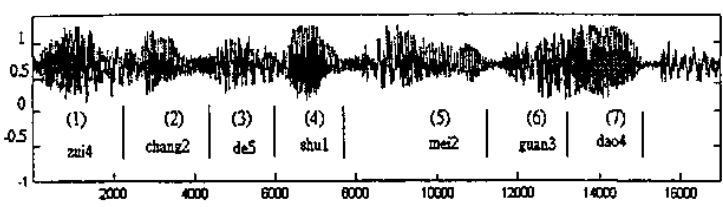

(b)

\begin{tabular}{|c|c|c|c|c|c|}
\hline$(1) /(2)$ & $(2) /(3)$ & $(3) /(4)$ & $(4) /(5)$ & $(5) /(6)$ & $(6) /(7)$ \\
\hline 1.04 & 1.43 & 0.71 & 0.63 & 1.17 & 1.10 \\
\hline
\end{tabular}

Fig. 3: (a) A segment of a speech utterance

(b) Duration ratios for adjacent syllable

tried to identify three features as described below to address such phenomenon.

1. Averaged pitch value over the syllable: If a syllable follows another syllable with a tone with a rising-end (tone 2 and tone 3 ), the pitch contour of the present syllable is naturally lifted up slightly so as to be closer to the end of the preceding pitch contour. Similarly, the pitch contour is naturally shifted down if the syllable follows another syllable with a tone with a falling end. For example, in Fig. 4(a) (tone sequence 1-3-4) and Fig. 4(b) (tone sequence 2-34), the vertical height of the pitch contour for the tone 3 syllable in Fig. 4(b) is higher than that in Fig. 4(a) by about $50 \mathrm{~Hz}$. Note that here all the pitch values have been normalized by a long term average [10]. This phenomenon may be modeled by a feature of averaged pitch value over the syllable. In other words, if a pitch contour is shifted down, this averaged value should be lower, etc.

2. Maximum pitch difference within the syllable: As mentioned above, the pitch contour of a syllable is often moved upward or downward, depending on the preceding syllable, but the pitch range of a speaker is more or less limited, or can't be too high or too low. In other words, if the pitch contour of a syllable is for some reason shifted down, but because the lowest pitch value can't be too low, as a result, the maximum pitch difference within the syllable has to be reduced to some degree. It's similar if the pitch contour is lifted up. For example, we can compare the pitch contour in Fig 4(c) (tone sequence 4-3-2) with that in Fig 4(b) (tone sequence 2-3-4). Although the middle syllable in Fig 4(b) and (c) are both of tone 3, the pitch contour of the middle syllable in Fig. 4(c) is apparently shifted lower as compared to that in Fig. 4(b) due to the difference in the context. As a result, the maximum pitch difference within the syllable is apparently smaller for the middle syllable in Fig. 4(c) as compared to that in Fig. 4(b). This is why the maximum pitch difference within the syllable is defined as a feature to characterize the context relationship among the tones. 

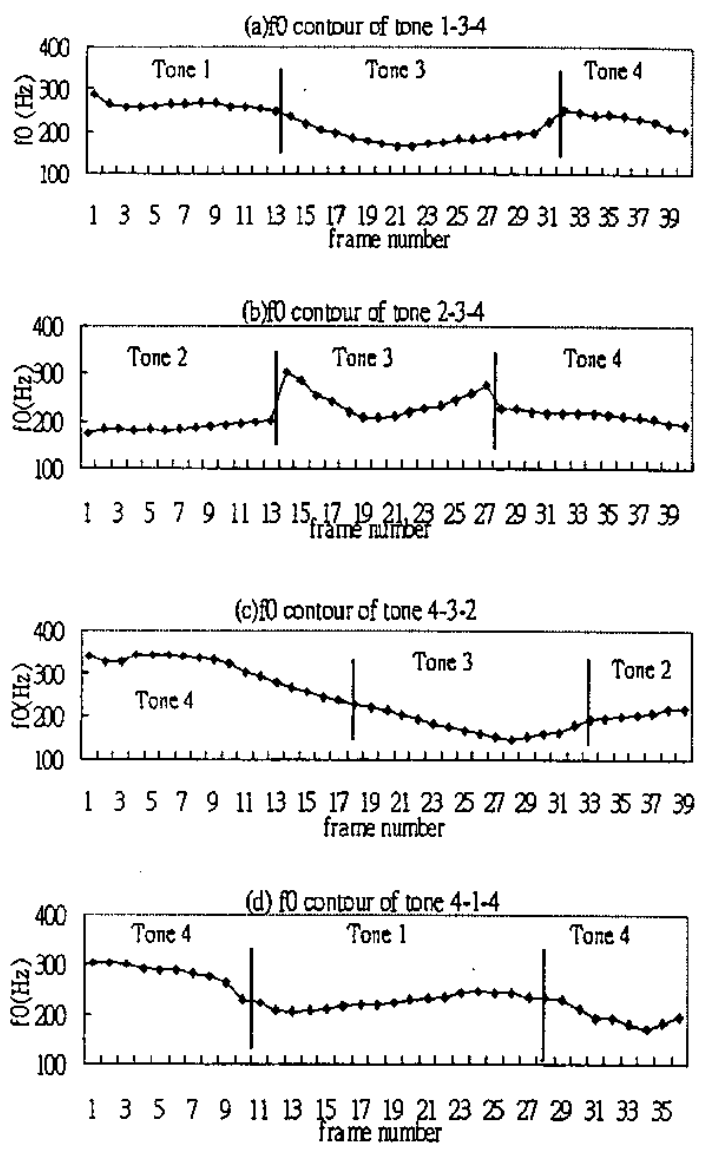

Fig. 4: Example pitch contours of (a) tone sequence 1-3-4 (b) tone sequence 2-3-4 (c) tone sequence 4-3-2 (d) tone sequence 4-1-4

3. Averaged slope of the pitch contour over the syllable: Take the tone 1 in Fig. 4(d) (tone sequence 4-1-4) as an example; in order to connect the low ending and high beginning of the tone 4 syllables on the two sides, the pitch contour of the tone 1 syllable has to be rising, exactly like a tone 2 , completely different from the typical pitch contour of a tone 1 , which is that with zero slope. Such cases actually happen quite frequently. So we tried to characterize such phenomenon with an extra feature of averaged slope of the pitch contour over the syllable.

\section{EXPERIMENTAL RESULTS}

\subsection{Pitch Extraction}

We used 60 sentences from HUB4 Mandarin broadcast news speech database which were spoken by 6 males and 6 females as the test set. The pitch value extracted manually was taken as the: reference values.

We compared five approaches for pitch extraction: the traditional autocorrelation method (AC), the subharmonic summation (SHS) [11], the method that uses AGR only (AGR), ESPS tools as well as the approach proposed here, AGR followed by IFAS (AGRIF). In addition to the clean speech case, four types of noise were added to the signals: Gaussian, car, restaurant, and babble noise, each at 15,5 and $2 \mathrm{~dB}$ of SNR. The error measurements used here are the gross pitch error (GPE, pitch error normalized by the reference pitch and multiplied by the frame energy), voiced-to-unvoiced (V-UV, percentage of voiced frames taken as unvoiced), and unvoiced-to-voiced (UV. $V$, percentage of unvoiced frames assigned with a pitch) error rates.

Fig. 5(a) shows the GPE for the five pitch extraction methods mentioned above tested under four different types of noisy environments: Gaussian, car, restaurant, and babble. For apparently the methods using Gaussian elementary functions, AGR and AGRIF perform under Gaussian noise much better than under other types of noises (e.g. comparing the cases of SNR of $2 \mathrm{~dB}$ in Fig. 5(a)). However, with other types of noise (car, restaurant, and babble), which are not necessarily Gaussian, the performance of AGR degrades seriously, but that of AGRIF doesn't. This is apparently because the transformation to IFAS domain effectively emphasizes the harmonic components. But in all cases regardless of the noise types or SNR values, the proposed approach (AGRIF) always performs the best. Table I lists the GPE value for the five pitch extraction methods under Gaussian noise as shown in the first part of Fig. 5(a), but divided into male/female speakers. It's clear that the new method has the best performance in all cases, except AGR is slightly better than AGRIF in clean speech case for male speakers.

Fig. 5(b), (c) shows the V-UV and UV-V performance under Gaussian noise at different values of SNR. Note that there is a natural tradeoff between V-UV and UV-V, i.e., a lower V-UV usually implies a higher UV-V. For example, the proposed method AGRIF and AGR both have the lowest V-UV error rates, but relatively higher UV-V error rates for lower SNR cases, while AC, SHS, and ESPS methods perform oppositely. Note that for the problem here, it is more important to detect the accurate pitch of a frame if it is voiced, while less harmful if an unvoiced frame is assigned a pitch, so by setting a lower threshold $\eta$ mentioned in section 2.2, the V-UV/UV-V error rates achieved by the proposed approach AGRIF is quite satisfactory in any case. Its V-UV error rates are always the lowest regardless of SNR in Fig. S(b), and its UV-V error rates are quite stable and always lower than AGR for all SNR values.

In a parallel set of experiments, HUB4 (Evltest) which includes 1146 sentences were tested. Just as the above, except the pitch values obtained by the ESPS tool were taken as the 


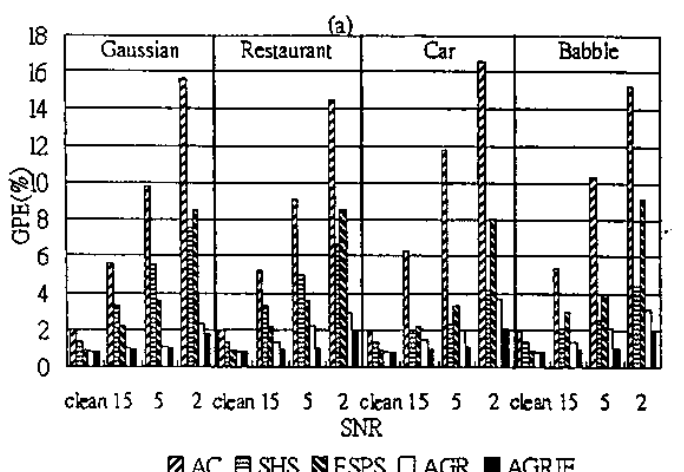

(b) V-UV(\%)
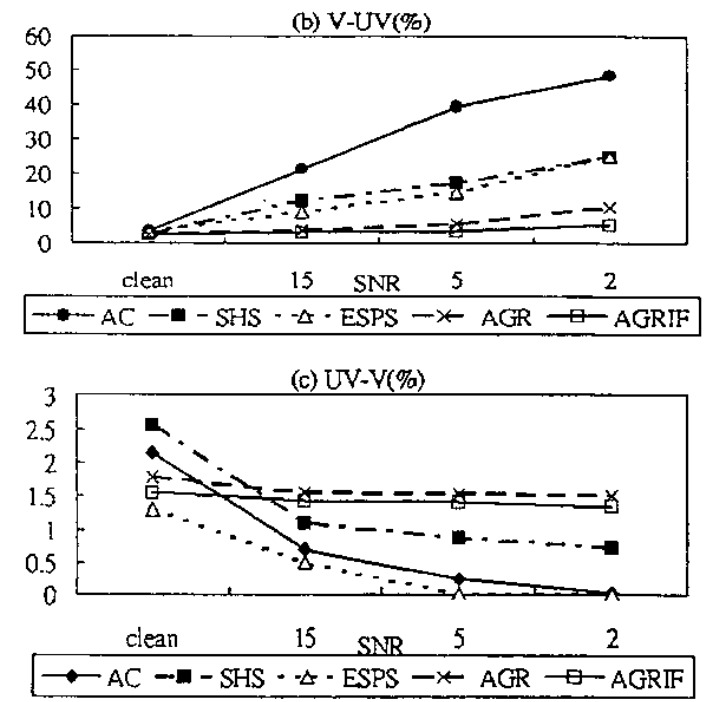

Fig. 5: (a) GPE for the five pitch extraction methods (AC, SHS, ESPS, AGR, AGRIF) under the noises (Gaussian, Restaurant, Car, Babble) (b) V-UV and (c) UV-V error rate for the five pitch extraction methods with Gaussian noise

reference values. The results and the general trends are very similar to those in Fig. 5 and Table 1, therefore not duplicated here.

\subsection{Tone Recognition}

For the tone recognition, we used HUB4 (Train) as the training corpus and HUB4 (Evltest) as the test corpus. We used the 13 MFCC parameters plus the logarithm pitch values and their first and second derivatives, thus a total of 42 features to train the tone models with different context conditions (175 HMM models). These models are to be used to perform the first-pass tone recognition. In addition, we used the four inter-syllabic features proposed in section 3 to train the tone models for different context conditions; each tone model has four Gaussian

\begin{tabular}{|c|c|c|c|c|c|}
\hline \multicolumn{7}{|c|}{ Male } \\
\hline SNR & AC & SHS & ESPS & AGR & AGRIF \\
\hline clean & 1.73 & 1.31 & 0.87 & 0.75 & 0.81 \\
\hline 15 & 6.11 & 2.95 & 2.04 & 1.01 & 0.99 \\
\hline 5 & 10.25 & 5.12 & 3.15 & 1.16 & 1.1 \\
\hline 2 & 19.62 & 6.89 & 7.92 & 2.38 & 1.98 \\
\hline
\end{tabular}

\begin{tabular}{|c|c|c|c|c|c|}
\hline \multicolumn{7}{|c|}{ Female } \\
\hline SNR & AC & SHS & ESPS & AGR & AGRIF \\
\hline clean & 2.21 & 1.01 & 1.13 & 0.88 & 0.85 \\
\hline 15 & 4.98 & 3.54 & 2.21 & 1.02 & 0.85 \\
\hline 5 & 8.12 & 5.36 & 3.92 & 1.05 & 1.01 \\
\hline 2 & 12.15 & 7.18 & 8.33 & 2.33 & 1.65 \\
\hline
\end{tabular}

Table 1: GPE for the five pitch extraction methods under Gaussian noise as shown in Fig 5(a), but divided by male/female speakers

mixtures. These models are to be used for the second-pass tone recognition. During the recognition gives the initial tones as well as the segmentation into syllables for the unknown utterances. This information is then used to evaluate the four inter-syllabic features for each syllable to be used in the second-pass recognition, which gives the final results. Because the pitch values vary with different speakers and the syllable position in the utterance, we always normalized the pitch values by the long term average [10], in both training and recognition. We also do these experiments under Gaussian noise at 15,5 , and $2 \mathrm{~dB}$ of SNR.

Table 2(a) is the confusion table for the result of the first-pass recognition under the clean environment without using the intersyllabic features, and Table 2(b) is the confusion table for the proposed method under the clean environment, after the secondpass recognition based on the inter-syllabic features. It can be found that the correct rate improvement for the neutral tone is very significantly, about $22 \%$ (57.2\% in Table 2 (a) to $79.2 \%$ in Table 2(b)), which shows that the duration ratio proposed here can effectively distinguish the neutral tone against the other tones. The confusion rate of incorrectly recognizing tone 3 as tone 2 is also very significantly reduced $(7.7 \%$ in Table 2 (a) to $5.4 \%$ in Table 2(b)). This is because the pitch contour of tone 3 is often very similar to tone 2 with certain context conditions, but such confusion is clarified to a good extent by the new intersyllabic features proposed here. In general, it can be found from Table 2(a), (b) that in most cases the tone recognition has been improved significantly.

Fig. 6 shows the averaged correct rate under Gaussian noise at different SNR of the recognition with and without inter-syllabic features. It is clear that even at low $(2 \mathrm{~dB}) \mathrm{SNR}$, the improvement of correct rate with inter-syllabic features can still be over 10 percent, which verifies the robustness of the proposed method. 
(a)

\begin{tabular}{|c|c|c|c|c|c|}
\hline & Tone 1 & Tone 2 & Tone 3 & Tone 4 & Tone 5 \\
\hline Tone 1 & 89.1 & 1.7 & 0.3 & 1.2 & 7.7 \\
\hline Tone 2 & 2.4 & 92.2 & 3.2 & 0.7 & 1.5 \\
\hline Tone 3 & 1.1 & 7.7 & 87.1 & 2.3 & 1.7 \\
\hline Tone 4 & 2.9 & 1.1 & 2.4 & 92.1 & 1.5 \\
\hline Tone 5 & 15.5 & 13.3 & 17.1 & 10.2 & 57.2 \\
\hline
\end{tabular}

(b)

\begin{tabular}{|l|c|c|c|c|c|}
\hline & Tone 1 & Tone 2 & Tone 3 & Tone 4 & Tone 5 \\
\hline Tone 1 & 92.5 & 2.1 & 1.4 & 1 & 3.01 \\
\hline Tone 2 & 1.1 & 95.8 & 1.5 & 0.5 & 1.1 \\
\hline Tone 3 & 0.8 & 5.4 & 91.2 & 1.9 & 0.7 \\
\hline Tone 4 & 2.5 & 0.6 & 1.7 & 94.8 & 0.4 \\
\hline Tone 5 & 9.5 & 6.5 & 6.2 & 5.1 & 79.2 \\
\hline
\end{tabular}

Table 2: The confusion table for (a) first-pass recognition without using the inter-syllabic features and (b) the final results after the second-pass recognition with the new set of features

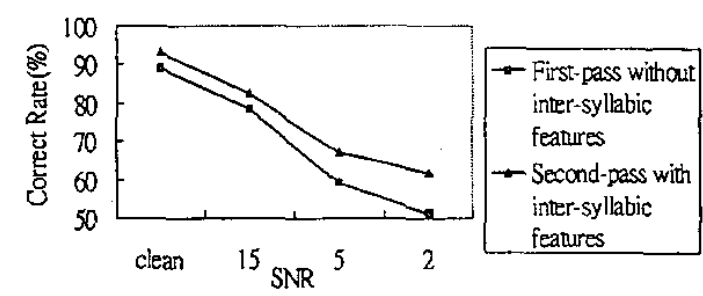

Fig. 6: Correct rate for with and without inter-syllabic features under Gaussian noise

\section{CONCLUSIONS}

In this paper, we present a set of four inter-syllabic features to recognize the tones in continuous Mandarin speech, and a new pitch extraction method by combining AGR and IFAS. Experimental results verified that the pitch extraction method is more robust under noisy environments than other methods, and the four new features can improve the tone recognition very well.

\section{REFERENCE}

[1] L. R. Rabiner, M. J. Cheng, A. E.Rosenberg, and C. A. McGonegal, "A comparative performance study of several pitch detection algorithms," IEEE Tras. Acous., Speech Audio Processing, vol. ASSP-24, pp.399-417, Oct. 1976.

[2] Tan Lee, Ching P.C., Chan L.W., Cheng Y.H., and Mak B. "Tone recognition of isolated Cantonese syllables," IEEE
Tras. Acous., Speech Audio Processing, Volume: 3 Issue: 3 , May 1995, Page(s): 204 -209

[3] Xin-Xian Chen, Chang-Nian Cai, Peng Guo and Ying Sun, "A Hidden Markov Model Applied to Chinese Four-Tone Recognition, ICASSP 1987, pp.797-800"

[4] Wu-Ji Yang, Jyh-Chyang Lee, Yuen-Chin Chang and Hsiao-Chuan Wang, "Hidden Markov Model for - Mandarin Lexical Recognition", IEEE Trans. on ASSP, Vol. 36, No7, July 1988, pp.988-992.

[5] Lin-Shan Lee "Voice dictation of Mandarin Chinese," Signal Processing Magazine, IEEE, Volume: 14 Issue: 4 , July 1997, Page(s): $63-101$

[6] J. S. Zhang and K. Hirose, "Tone Recognition of Chinese Continuous Speech Using Tone Critical Segments," Proc. of Eurospeech'99, Budapest, Hungary, Sept. 1999, pp.879-882.

[7] D.J. Liu and C. T. Lin, "Fundamental Frequency Estimation Based on the Joint Time-Frequency Analysis of Harmonic Spectral Structure" IEEE Trans. Speech Audio Processing, vol. 9, NO. 6, Sept. 2001

[8] Shie Qian and Dapang Chen, "Joint Time-Frequency Analysis" Prentice Hall, 1996

[9] Abe T, Kobayashi T., and Imai S." Harmonics tracking and pitch extraction based on instantaneous frequency," ICASSP-95., Volume: 1, 9-12 May 1995. Page(s): 756. 759 vol.1

[10] Hank Chang-Han Huang; Seide, F.," Pitch tracking and tone features for Mandarin speech recognition," ICASSP '00. Proceedings. Volume: 3, 5-9 June 2000. Page(s): $1523-1526$ vol. 3

[11] Xuejing Sun," Pitch determination and voice quality analysis using subharmonic to harmonic ratio", ICASSP '02. Volume: 1 , 13-17 May 2002 . Page(s): I-333 -I-336 vol.1 